\title{
ILP FORMULATION OF GROOMING OVER WAVELENGTH ROUTING WITH PROTECTION
}

\author{
Tibor Cinkler \\ High-Speed Networks Laboratory, Department of Telecommunications \& Telematics \\ Budapest University of Technology \& Economics, Budapest, Hungary \\ cinkler@ttt-atm.ttt.bme.hu
}

\begin{abstract}
An efficient and general graph-theoretic model (the Wavelength-Graph (WG)) has been proposed which enables Integer Linear Programming (ILP) formulation of static Routing and Wavelength Assignment (RWA) problems in Multihop Wavelength Routing (WR) Dense Wavelength Division Multiplexing (DWDM) Networks.

The topology of the physical layer, the type of each node (e.g., OADM, OXC or EXC), the number of available wavelengths per link and the capacity of each wavelength-channel are assumed given with the aggregated traffic demand of each node-pair. The output of the optimisation is the system of wavelength-paths, lightpaths and semilightpaths.

The objective of the optimisation is to reduce resource usage at upper (electrical) layers, subject to constrained amount of capacity of each wavelength and limited number of wavelengths.
\end{abstract}

\section{INTRODUCTION}

WDM has been introduced to increase the transmission capacity of existing optical links. Instead of using one several transmitter and receiver pairs were used over the same fibre at different wavelengths forming independent channels and over-bridging the speed limitations of electronics. It has been soon recognised that the switching decision can be made according to the incoming wavelength without any processing of the data stream. In single-hop DWDM based All-Optical Networks $(\mathrm{AON})$ a wavelength is assigned to a connection in such a way that each connection (wavelength) is handled (switched) in the optical domain without any electrical conversion during the transmission [1]. This will be referred to as a wavelength-path. Wavelength (WL) reuse is allowed 
in parts of the network where that WL was not used. This WDM AON would require a huge number of different WLs. The technology sets the limit nowadays to around 160 different WLs per fibre in the $1550 \mathrm{~nm}$ window with $25 \mathrm{GHz}$ (about $0.2 \mathrm{~nm}$ ) spacing in the flat operating gain band (1530-1560 nm) of the present Erbium-Doped Fibre Amplifiers (EDFA) according to the recently completed ITU-T Recommendation G.692. For this reason WL conversions are needed. The most expensive way is to make optical WL conversion which ensures transparency of the network. Then, the end-to-end channel will be referred to as a lightpath. Simpler and cheaper method is to do first opto-electrical conversion, electrical space-switching and then electro-optical conversion. The endto-end connection will then use a semi-lightpath. This is the idea for realising so called opaque networks, where systems using different sets of WLs are to be interconnected. The optimisation method proposed in this paper can be applied to all, wavelength-paths, lightpaths and semi-lightpaths.

It has been shown in [2] that the required number of different wavelengths per fibre for networks with and without WL conversion capability is about the same. In general, for networks of practical size, the number of available wavelengths is lower by a few orders of magnitude than the number of connections to be established. The only solution here is to join some of the connections to fit into the available wavelength-links. This is referred to as traffic grooming). This can be done at electrical layer only since re-multiplexing, i.e., time division multiplexing is required. For this reason, taking not only the optical, but both, optical and electrical layer into account when configuring the system is demanded.

Many excellent papers deal with design, configuration and optimisation of WDM Networks. See, e.g., [2-8]. The widely accepted approach is to decompose the problem to the following sub-problems in given order. First, determine the virtual topology (route the light-paths); second, assign a wavelength to each light-path (WA); and third, route the traffic over the light-paths.

In [3] a heuristic (greedy) algorithm is proposed for WA followed by routing over established light-paths. In [4] is defined a bound for carried traffic in all-optical networks and shown that the proposed heuristic Routing and Wavelength Assignment (RWA) algorithm gives results very close to this bound. A performance study has been carried out. In [5] mathematical formulation of the design problem is given along with heuristics for solving the sub-problems relaxing some of the constraints one-by-one. In [2] there is also given a heuristic algorithm, and is showed that networks with and without wavelength converters require about the same number of wavelengths. A hybrid solution is also proposed where 
wavelengths are electrically regenerated in some specific nodes. In [6] multi-commodity flow model with randomised rounding is applied followed by graph colouring algorithms. In [7] an algorithm is given for light-path routing by transforming the network to a special structure called wavelength graph (Although we will use the same term the structure covered by it differs significantly). In this graph costs are assigned to edges and shortest path algorithms are run. The optimality of the algorithm is also proved. In [8] design principles of Optical Networks are explored. The design is formulated as an optimisation problem with two objectives to be solved by heuristics. The (weighted) average delay trough the network is minimised, while the total carryable traffic over the network is maximised. In [9] the extended layered graph is used which is a bit similar to our model, but not flexible enough. In [10] the optical path routing strategies for WDM networks are investigated. The performance of networks with and without wavelength converters is evaluated. In [11] is described a method for planning WDM layer for carrying ATM traffic over it with the survivability constraint using Tabu Search. In [12] a reconfigurable OADM is demonstrated. In [13] hitless reconfiguration of WDM networks is investigated. [14] gives a performance evaluation and comparison of WDM networks with and without wavelength interchange capability. [15] proposes an algorithm for simultaneous Routing and Wavelength Assignment on a Path Graph. Channel capacities are not taken into account. In [16] an exact linear programming formulation is presented and the "closest" virtual topology is chosen for reconfiguration. [17] investigates the performance of partial reconfiguration on a SDM/WDM architecture. [18] proposes a model and algorithm for finding the globally optimal WL assignment with high probability using generally applicable heuristics for global optimisation. In [19] Mixed Integer Linear Programming (MILP) formulation of the static RWA problem is given for the case without WL changers as a minimax problem. The MILP formulation is then relaxed to LP and solved, followed by rounding.

Our subject is to configure the light-path system optimally without separating the network-layers. This improves the quality of results, but on the other hand the complexity of the problem grows.

As the optimisation result we decrease the traffic to be processed and carried in the electrical domain over-bridging the speed limits of electronics. Since a considerable part of the load of electrical, e.g., SDH, ATM or MPLS switches is undertaken by the optical switches much larger networks with higher loads can be realised by the current technology offering better granularity and using optimally any limited number of WLs. 
In Section 2 we present the model of the network with different nodetypes. In Section 3 we formulate the problem first informally, and then formally as an Integer Linear Program (ILP) for both, directed and undirected graph models. In Section 4 th protection alternatives are given, for protection in both, upper electrical layer and lower wavelength layer. Section 5 adds some comments on the problem, while in Section 6 solution alternatives are proposed.

\section{THE WAVELENGTH GRAPH (WG)}

The task was to provide a general model for configuration of WDM networks with different types of nodes and arbitrary topologies. Although the most popular topology is ring or interconnected rings, the model must be able to handle any specific or mesh topology. The nodes can also be quite different: Optical Add-and-Drop Multiplexers (OADM), Optical Cross-Connects (OXC) with full or limited (optical or opto-electrical) WL conversion or even an Opto-Electrical Cross Connect (OEXC). The protection strategies can also be quite different. All these aspects are taken into account in the proposed model. First the link model is described followed by models of different nodes. In this section we assume that all traffic demands are bidirectional and symmetrical. In this case the network can be modeled by an undirected graph. The model can be simply generalised for un-symmetrical demands, by using directed graphs. In later case the model is more complex and for this reason the algorithms will run slower.

\subsection{MODEL OF LINKS}

A network consists of nodes, and links connecting the nodes. This can be modeled by a graph: a node is a vertex and a link is an edge. Having multiple WLs we will represent a WL of a link as an edge in the graph of wavelengths according to Figure 1 for the network proposed in [20]. To prioritise filling up WLs one-by-one we can assign slightly different weights to different channels of one link. For example, edges representing WL1, WL2 and WL3 will have weights 101, 102 and 103 respectively.

\subsection{MODEL OF NODES}

A node is modeled by a subgraph. The subgraph-nodes are the certain WLs at the switch-ports, while the weighted edges represent the costs of transitions, terminations, conversions, etc. There are different types of nodes. Models of nodes differ for these. Here will be shown some examples. In similar manner a model can be derived for any additional 


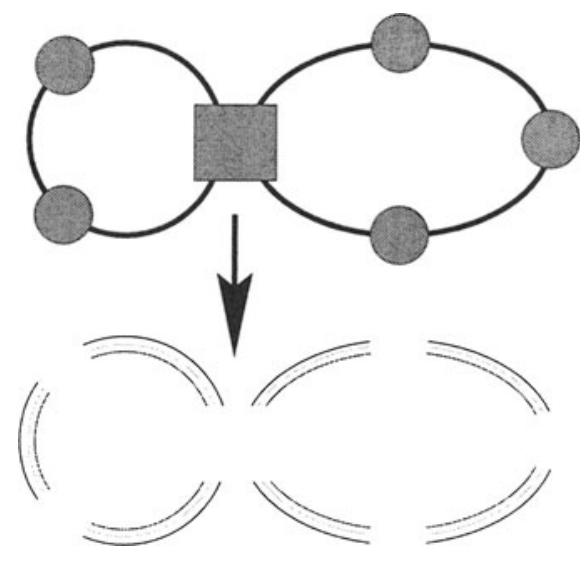

Figure 1 Modeling edges.

node-type. The models proposed here are similar to those described in [21], but those were used for setting up connections one-by-one using shortest path algorithms, while here is the emphasis on global simultaneous configuration requiring special node-models.

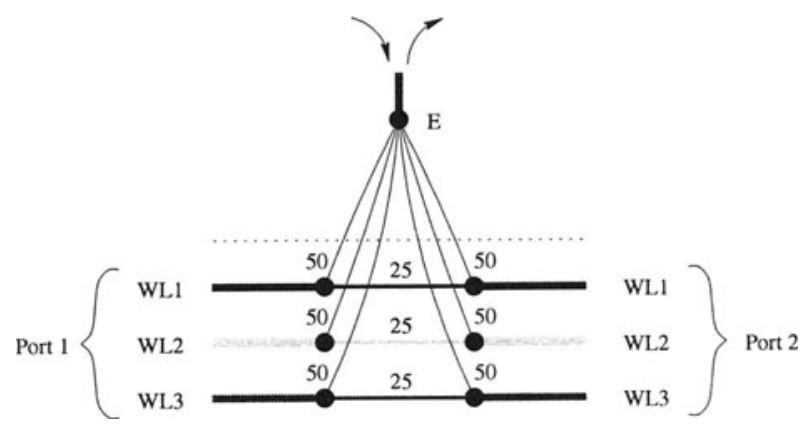

Figure 2 Model of OADM Nodes.

Optical Add-and-Drop Multiplexer: OADM. The OADM Nodes have in general two bi-directional ports (4 fibres). Their function is either to transmit a WL channel or to terminate it and usually they do not allow WL-conversion. These devices are capable to add or drop wavelength channels. 
The weights assigned to edges representing termination (e.g., 50) are higher than weights of transition (e.g., 25), because transition is preferred to termination. According to the proposed model (Figure 2) the traffic streams can enter or exit the OADM crossing vertex $\mathrm{E}$ or can be even re-multiplexed.

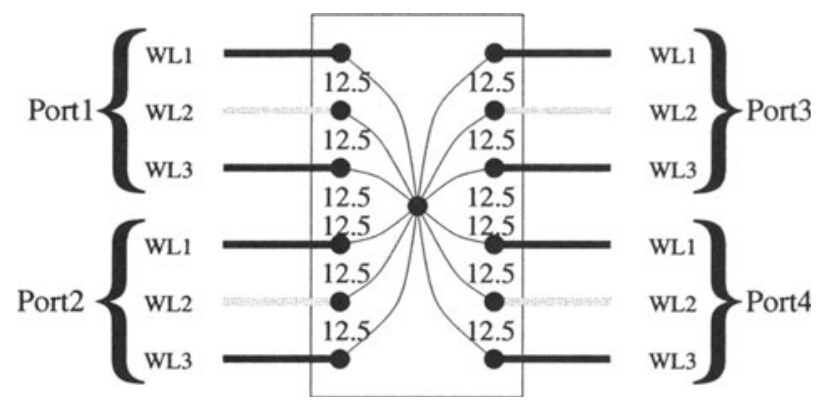

Figure 3 Model of OEXC Nodes.

Optical Cross-Connect with Electrical Core: OEXC. In the model shown in Figure 3 each pair of nodes should be connected by an edge. All edges should have equal weights. Instead of connecting all pairs using $n \times n$ edges we use $n$ edges and one node. This simplifies the model. Each incoming channel is converted to electrical domain switched by a space-switch and again converted to the optical domain to arbitrary WL. Each termination, transition or WL change of a light-path has the same cost (e.g., 25). Therefore all edges have the same weight (e.g., $25 / 2)$.

Optical Cross-Connect with All-Optical Core: OXC. An optical Cross-Connect has more than two ports, e.g., four bi-directional ports according to Figure 1. In an OXC a light-path can make transition to any output port which supports that WL, and that WL is not yet used. This OXC type (without WL change capability) will be referred to as simple OXC (see Figure 4). In this case one incoming channel can exit at any of the remaining output ports where that WL is supported and not yet used.

In some OXC devices WL translation (change) is also supported. This node will be called OXC. Its model is showed in Figure 5. For this node any incoming channel can exit at one of 11 remaining channels. Now there are 3 possibilities for light-path transition since there are channels 


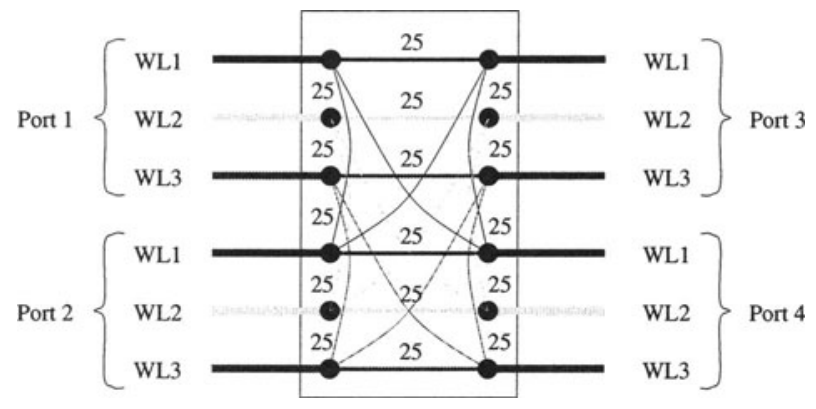

Figure 4 Model of a Simple Optical Cross-Connect Node (without WL conversion)

of the same wavelength on all ports in our example. For WL change there are 8 possibilities. It has higher cost (e.g., $2 \times 50=100$ ) than the WL transition. WL change is modeled by conducting the traffic stream through node $\mathrm{E}$.

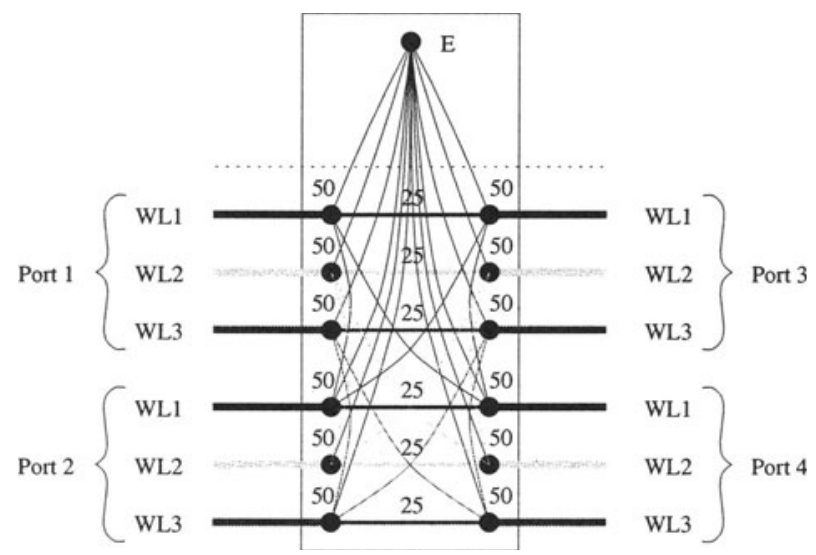

Figure 5 Model of an Optical Cross-Connect Node (with WL conversion)

In some cases the traffic stream termination is also among the functions of an OXC. In that case the model does not need any change. The only difference will be that there will be some traffic offered to that OXC node which can be modeled by offering traffic to node $\mathrm{E}$ and considering it as an end-node. In this case traffic-stream re-multiplexing capability is also required. 
Modeling opto-electro-optical conversions, multiplexing and re-multiplexing. If we want to differentiate the simple wavelengthchange from the electrical signal re-multiplexing a more complex model is needed. An example has been shown in Figure 6 for an OADM node for simplicity reasons, which can be extended to any other node-type. As can be seen node $\mathrm{E}$ has been substituted by a fully connected sub-graph. In this case assigning costs to internal edges the costs of wavelengthchange and signal re-multiplexing can be differentiated.

All-Optical WL conversion is not supported by all OXCs. Therefore the optical signal is terminated and passed to the electrical layer where space switching or space switching with time switching (re-multiplexing) is done and then the resulting electrical signal passed back to the optical layer.

In cross connects three levels of cross-connecting and switching are to be differentiated:

- WL transition - This is done by the optical layer without any processing. This is the preferred and cheapest function. The signal can bypass the electrical layer using lightpaths of the optical layer.

- WL translation - It can be carried out by optical WL shifters, or by opto-electrical conversion, space-switching and electro-optical conversion. It is more expensive than the previous function, but still cheaper than the next one. Here is the switching very simple and no traffic stream processing is needed.

- multiplexing and re-multiplexing - In a larger WDM transport network there are considerably less available WLs per fibre than it would be needed for full interconnection of the end nodes by singlehop lightpaths. For this reason some of the traffic streams have to be multiplexed along a lightpath, i.e., in some cases the lightpath termination is not a traffic stream termination. In these cases time-division re-multiplexing is needed. In this case the part of the equipment performing the Time Division Multiplexing is able to add traffic to that node or to drop it. In this paper we will not take this capability into account.

\section{PROBLEM FORMULATION}

It is algorithmically very complex to obtain globally optimal solution for the global simultaneous routing and wavelength assignment problem. (The problem can be expressed as well as configuration of the lightpath system of a WDM network.) This problem very likely belongs to the class 


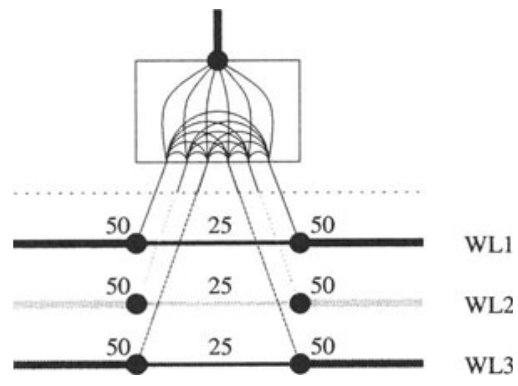

Figure 6 Modeling opto-electro-optical conversions and re-multiplexing: the complex model

of NP-hard problems [22], because its sub-problem, the Static Lightpath Establishment (SLE) has been shown to be NP-hard [23].

The task was to find a shortest path in the obtained graph (which is built up of the link and node models) between all pairs of nodes simultaneously. There are alternatives for choosing the objective of the optimisation, e.g.,:

1. Minimise the total number of used WLs per fibre.

2. Decrease the total amount of used resources at the optical layer.

3. Decrease the total amount of used resources and processing at the electrical layer.

4. Decrease the total amount of lost traffic. It is also possible to introduce a scale-up factor for all traffic demands. For example scaling up all traffic demands by $10 \%$ the network should still work properly.

5. Minimise the number of WL conversions in total and for each path.

Our objective function will optimise 3. and 5. simultaneously, as will be discussed.

\subsection{ILP FORMULATIONS FOR DIRECTED GRAPHS}

The above described problem can be formulated as an Integer Linear Program using the proposed model. For this purpose we will first formulate the problem for the directed graph model. This formulation has slight similarities with Minimal Cost Multicommodity Flow (MCMCF) problem formulation [24].

Let $\mathrm{D}(\mathrm{V}, \mathrm{A}, \mathrm{C})$ be a directed graph (digraph) where $V$ is the set of vertices, $A=\{(i, j) \in A, i, j \in V\}$ is the set of arcs (directed edges) and $C=\left\{c_{i j} \in R^{+},(i, j) \in A\right\}$ is the cost of using arc $(i, j)$. Set $O=$ $\left\{o\left(s^{o}, t^{o}, b^{o}\right): s^{o}, t^{o} \in V ; b^{o} \in R^{+}\right\}$is the set of all demands characterised 
by its source $s^{o}$ sink $t^{o}$ and bandwidth requirement $b^{o} . \quad V_{E} \subset V$ is the set of vertices representing electrical nodes capable of performing grooming, i.e., time division multiplexing. These nodes are denoted by ' $\mathrm{E}$ ' in Figures 2 and 5. $V_{i}^{+}=\{k:(i, k) \in A\}$ and $V_{i}^{-}=\{k:(k, i) \in A\}$ denote all arcs entering and leaving node $i$ respectively.

The number of the wavelength channels is assumed to be equal for all fibers, and each link consists of two fibers carrying information in opposite directions. Without loss of generality for simplicity reasons the capacity $B$ of each wavelength channel is assumed to be equal as well.

Variable $x_{i j}^{o} \in\{0,1\}$ denotes flow of commodity $o$ in arc $(i, j)$, while variable $y_{i j} \in\{0,1\}$ indicates, whether lighpath spanning between $i$ and $j$ is used (1) or not (0). For illustration see Figure 7, where the used internal light-links are denoted by solid lines.

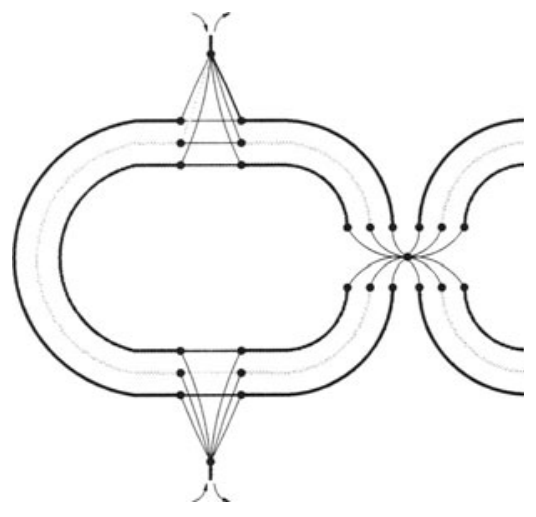

Figure 7 The graph model of a part of the network shown in Figure 1 with 2 OADM and 1 OEXC node.

Objective:

$$
\begin{aligned}
\text { minimise } & \left(\alpha C_{E}+(1-\alpha) C_{O}\right) \\
\text { where } & 0 \leq \alpha \leq 1 \\
\text { while } & C_{E}=\sum_{(i, j) \in A, i \in V_{E} \vee j \in V_{E}} \sum_{o \in O} c_{i j} b^{o} x_{i j}^{o} \\
\text { and } & C_{O}=\sum_{(i, j) \in A, i \notin V_{E} \wedge j \notin V_{E}} \sum_{o \in O} c_{i j} y_{i j}^{o}
\end{aligned}
$$

Subject to constraints: 


$$
\begin{gathered}
\sum_{\forall o \in O} x_{i j}^{o} b^{o} \leq B \\
x_{i j \leq y_{i j}}^{o} \quad \forall(i, j) \in A \\
y_{i j} \leq \sum_{\forall o \in O} x_{i j}^{o} \quad \forall(i, j) \in A \\
\sum_{\forall j \in V_{i}^{+}} x_{j i}^{o}-\sum_{\forall k \in V_{i}^{-}} x_{i k}^{o}=\left\{\begin{aligned}
-1 & \text { if } i=s^{o} \\
0 & \text { if } i \in V \backslash\left\{s^{o}, t^{o}\right\} \\
1 & \text { if } i=t^{o}
\end{aligned}\right. \\
\text { for all nodes } i \in V \text { and commodities } o \\
y_{j i}=\sum_{\forall k \in V_{i}^{-}} y_{i k} \quad \forall i \in V \backslash V_{E}, \forall j \in V_{i}^{+}
\end{gathered}
$$

Variables:

$$
\begin{array}{ll}
x_{w}^{o} \in\{0,1\} \quad \forall(i, j) \in A, \quad \forall o \in O \\
y_{i j} \in\{0,1\} \quad \forall(i, j) \in A
\end{array}
$$

The objective is to minimise the number of hops for each traffic demand weighted by the required capacity of that traffic stream and by the cost of using those light-links subject to the following constraints. Constraint (5) states that the amount of traffic using a light-link may not exceed the capacity B of that light-link.

Constraint (8) ensures that traffic-streams are to be terminated at end-nodes and the traffic flows must be conserved at each non-end node.

Constraints (6) and (7) guarantee that traffic streams may use available light-paths only, and a light-path will be established only if it is needed for carrying a traffic flow. Ninth constraint expresses that a light-path can not branch.

The last two constraints (10) and (11) mean that both variables can take values 0 or 1 only.

The optimisation will result in a single-hop configuration (Wavelengthpaths) whenever possible or in a multihop configuration with as few WL translations and re-multiplexing as possible, i.e., the largest possible part of the load of the electrical layer will be overtaken by the optical layer.

If the aim was to decrease the number of used WLs in total, the value of $\alpha$ should be strictly less than 1, possibly as close to 0 as possible. Then constraint (7) can be avoided. 


\subsection{ILP FORMULATIONS FOR UNDIRECTED GRAPHS}

To decrease the number of both, variables and constraints in this Sub-section the undirected graph model will be used.

Let $\mathrm{U}(\mathrm{V}, \mathrm{E}, \mathrm{C})$ be the undirected graph (ugraph) where $V$ is the set of vertices, $E=\{(i, j) \in E, i, j \in V\}$ where $(i, j)=(j, i)$ is the set of (undirected) edges and $C=\left\{c_{i j} \in R^{+},(i, j) \in E\right\}$ is the cost of using edge $(i, j)$. Set $O=\left\{o\left(s^{o}, t^{o}, b^{o}\right): s^{o}, t^{o} \in V ; b^{o} \in R^{+}\right\}$is the set of all demands characterised by its two ends $s^{o}$ and $t^{o}$ and bandwidth requirement $b^{\circ} . V_{E} \subset V$ is the set of vertices representing electrical nodes capable of performing grooming, i.e., time division multiplexing. $V_{i}$ is the set of neighbour-nodes of node $i$ (nodes adjacent to $i$ ) defined as $V_{i}=\{j:(i, j) \in E\}$, remember, that $(i, j)=(j, i)$.

As for the directed case the number of the wavelength channels is assumed to be equal for all fibers, and each link consists of two fibers carrying information in opposite directions. Without loss of generality for simplicity reasons the capacity $B$ of each wavelength channel is assumed to be equal as well.

Variable $x_{i j}^{o} \in\{0,1\}$ denotes flow of commodity $o$ in edge $(i, j)$, while variable $y_{i j} \in\{0,1\}$ indicates, whether lighpath spanning between $i$ and $j$ is used (1) or not (0).

Objective:

$$
\begin{aligned}
\text { minimise } & \left(\alpha C_{E}+(1-\alpha) C_{O}\right) \\
\text { where } & 0 \leq \alpha \leq 1 \\
\text { while } & C_{E}=\sum_{(i, j) \in E, i \in V_{E} \vee j \in V_{E}} \sum_{o \in O} c_{i j} b^{o} x_{i j}^{o} \\
\text { and } & C_{O}=\sum_{(i, j) \in E, i \notin V_{E} \wedge j \notin V_{E}} \sum_{o \in O} c_{i j} y_{i j}^{o}
\end{aligned}
$$

Subject to constraints:

$$
\begin{aligned}
\sum_{\forall o \in O} x_{i j}^{o} b^{o} \leq B & \forall(i, j) \in E \\
x_{i j}^{o} \leq y_{i j} & \forall(i, j) \in E, \quad \forall o \in O \\
y_{i j} \leq \sum_{\forall o \in O} x_{i j}^{o} & \forall(i, j) \in E \\
\sum_{\forall j \in V_{i}} x_{i j}^{o}=1 & \forall i \in V_{E}, \quad \forall o \in O
\end{aligned}
$$




$$
\begin{array}{ll}
\sum_{\forall j \in V_{i}} x_{i j}^{o}=2 z_{x i}^{o} \quad & \forall i \in V \backslash V_{E}, \quad \forall o \in O \\
\sum_{\forall j \in V_{i}} y_{i j}=2 z_{y i} & \forall i \in V \backslash V_{E}
\end{array}
$$

Variables:

$$
\begin{array}{ll}
x_{w}^{o} \in\{0,1\} & \forall(i, j) \in E, \quad \forall o \in O \\
y_{i j} \in\{0,1\} & \forall(i, j) \in E \\
z_{x i}^{o} \in\{0,1\} & \forall i \in V \backslash V_{E}, \quad \forall o \in O \\
z_{y i} \in\{0,1\} & \forall i \in V \backslash V_{E}
\end{array}
$$

where the variable $z$ has been used for emulating the condition "equal to 0 or 2 "

As in the case for directed graphs the objective is to minimise the number of hops for each traffic demand weighted by the required capacity of that traffic stream and by the cost of using those light-links subject to the following constraints. Constraint (16) states that the amount of traffic using a light-link may not exceed the capacity B of that light-link.

Constraints (19) and (20) ensure that traffic-streams are to be terminated at end-nodes and the traffic flows must be conserved at each non-end node.

Constraints (17) and (18) guarantee that traffic streams may use available light-paths only, and a light-path will be established only if it is needed for carrying a traffic flow. 21th constraint expresses that a lightpath can not branch.

The last four constraints (23) - (26) mean that all variables can take values either 0 or 1 .

The problem formulated here is the same as it was for the directed graph, with only difference, that it is less complex, i.e., needs less variables and constraints.

If the aim was to decrease the number of used WLs in total, the value of $\alpha$ should be strictly less than 1, possibly as close to 0 as possible. Then constraint (18) can be avoided.

\section{PROTECTION}

Here we assume dedicated (i.e., either $1+1$ or $1: 1$ ) protection. The aim is to protect all demands either at the electrical or at the optical layer by using link- or node-disjoint working and protection path for each demand. ILP formulation of these cases follows. 


\subsection{ILP FORMULATION OF PROTECTION AT UPPER LAYERS}

Protection at upper layers means that the end-to-end connections at the uppermost layer have to be protected by configuring a protection path at the uppermost layer for each working path which is physically disjoint of the working one. That is the two paths may not use a common cable or node at the same geographical location.

Link Disjoint Case. To formulate the Link Disjoint case we have to introduce sets of edges $(i, j)$ which share a common optical cable or even a duct. Let $L$ be the set of all physical links $l$. Then $L=\{l: l \subset$ $V, \forall(i, j) \in l$ belong to the same physical link $l\}$. Here we will continue using directed graphs, however, as shown in Section 3 we can reformulate it for directed graphs whenever needed.

We need a set of new variables. Instead of $x_{i j}^{o}$ we will use $x 1_{i j}^{o}$ and $x 2_{i j}^{o}$ for indicating working and protection flow of demand $o$ over edge $(i, j)$. In this way we have doubled the number of variables, and slightly increased the number of constraints as well.

Now the formulation is as follows:

Objective:

$$
\begin{aligned}
\text { minimise } & \left(\alpha C_{E}+(1-\alpha) C_{O}\right) \\
\text { where } & 0 \leq \alpha \leq 1 \\
\text { while } & C_{E}=\sum_{(i, j) \in A, i \in V_{E} \vee j \in V_{E}} \sum_{o \in O} c_{i j} b^{o}\left(x 1_{i j}^{o}+x 2_{i j}^{o}\right) \\
\text { and } & C_{O}=\sum_{(i, j) \in A, i \notin V_{E} \wedge j \notin V_{E}} \sum_{o \in O} c_{i j} y_{i j}^{o}
\end{aligned}
$$

Subject to constraints:

$$
\begin{aligned}
\sum_{\forall o \in O}\left(x 1_{i j}^{o}+x 2_{i j}^{o}\right) b^{o} \leq B & \forall(i, j) \in A \\
x 1_{i j}^{o}+x 2_{i j}^{o} \leq y_{i j} & \forall(i, j) \in A, \quad \forall o \in O \\
y_{i j} \leq \sum_{\forall o \in O}\left(x 1_{i j}^{o}+x 2_{i j}^{o}\right) & \forall(i, j) \in A \\
\sum_{\forall(i, j) \in l}\left(x 1_{i j}^{o}+x 2_{i j}^{o}\right) \leq 1 & \forall l \in L, \quad \forall o \in O
\end{aligned}
$$




$$
\begin{gathered}
\sum_{\forall j \in V_{i}^{+}} x 1_{j i}^{o}-\sum_{\forall k \in V_{i}^{-}} x 1_{i k}^{o}=\left\{\begin{aligned}
-1 & \text { if } i=s^{o} \\
0 & \text { if } i \in V \backslash\left\{s^{o}, t^{o}\right\} \\
1 & \text { if } i=t^{o}
\end{aligned}\right. \\
\text { for all nodes } i \in V \text { and commodities } o
\end{gathered}
$$

$$
\begin{gathered}
\sum_{\forall j \in V_{i}^{+}} x 2_{j i}^{o}-\sum_{\forall k \in V_{i}^{-}} x 2_{i k}^{o}=\left\{\begin{aligned}
-1 & \text { if } i=s^{o} \\
0 & \text { if } i \in V \backslash\left\{s^{o}, t^{o}\right\} \\
1 & \text { if } i=t^{o}
\end{aligned}\right. \\
\text { for all nodes } i \in V \text { and commodities } o
\end{gathered}
$$

$$
y_{j i}=\sum_{\forall k \in V_{i}^{-}} y_{i k} \quad \forall i \in V \backslash V_{E}, \forall j \in V_{i}^{+}
$$

Variables:

$$
\begin{array}{rlrl}
x 1_{w}^{o} \in\{0,1\} & \forall(i, j) \in A, & \forall o \in O \\
x 2_{w}^{o} \in\{0,1\} & \forall(i, j) \in A, \quad \forall o \in O \\
y_{i j} \in\{0,1\} & \forall(i, j) \in A &
\end{array}
$$

In this formulation constraint (34) is that one which ensures diversity (i.e., link disjointness) of working and protection paths. Note, that we have implicitly assumed that arcs between the same pair of nodes of different direction $(\mathrm{i}, \mathrm{j})$ and $(\mathrm{j}, \mathrm{i})$ belong to the same set $l$. This constraint allows to simply substitute $x_{i j}^{o}$ by $\left(x_{i j}^{o}+x_{i j}^{o}\right)$ in all constraints, except for (35) and (36).

Node Disjoint Case. If we want to make the protection and working paths not only link- but node-disjoint as well then instead of (34) we should write any of the following constraints:

$$
\begin{array}{ll}
\sum_{\forall i \in n \backslash V_{E}^{o}} \sum_{\forall j \in A_{i}^{+}}\left(x 1_{i j}^{o}+x 2_{i j}^{o}\right) \leq 1 & \forall n \in N, \quad \forall o \in O \\
\sum_{\forall i \in n \backslash V_{E}^{o}} \sum_{\forall j \in A_{i}^{-}}\left(x 1_{i j}^{o}+x 2_{i j}^{o}\right) \leq 1 & \forall n \in N, \quad \forall o \in O
\end{array}
$$

In this case constraints (35) and (36) can be written as one constraint, i.e., instead of them we should write

$$
\sum_{\forall j \in V_{i}^{+}}\left(x 1_{j i}^{o}+x 2_{j i}^{o}\right)-\sum_{\forall k \in V_{i}^{-}}\left(x 1_{i k}^{o}+x 2_{i k}^{o}\right)=\left\{\begin{aligned}
-1 & \text { if } i=s^{o} \\
0 & \text { if } i \in V \backslash\left\{s^{o}, t^{o}\right\} \\
1 & \text { if } i=t^{o}
\end{aligned}\right.
$$

for all nodes $i \in V$ and commodities $o(43)$ 


\subsection{ILP FORMULATION OF PROTECTION AT THE DWDM LAYER}

Protection at the DWDM layer means that the end-to-end connections at the uppermost layer have to be protected by configuring a protection wavelength path for each working wavelength path which is physically disjoint of the working one. That is the two paths may not use a common cable or a node at the same geographical location.

Link Disjoint Case. In this case we should substitute all variables $y_{i j}$ by $y 1_{i j}$ and $y 2_{i j}$ and rewrite the objective functions and constraints in way to ensure, that for each working wavelength-path there should be a disjoint protection one.

Objective:

$$
\begin{aligned}
\underset{\text { minimise }}{\text { where }} & \left(\alpha C_{E}+(1-\alpha) C_{O}\right) \\
\text { while } & C_{E}=\sum_{(i, j) \in A, i \in V_{E} \vee j \in V_{E}} \sum_{o \in O} c_{i j} b^{o} x_{i j}^{o} \\
\text { and } & C_{O}=\sum_{(i, j) \in A, i \notin V_{E} \wedge j \notin V_{E}} \sum_{o \in O} c_{i j}\left(y 1_{i j}^{o}+y 2_{i j}^{o}\right)
\end{aligned}
$$

Subject to constraints:

$$
\begin{gathered}
\sum_{\forall o \in O} x_{i j}^{o} b^{o} \leq B \quad \forall(i, j) \in A \\
x_{i j \leq y_{i j}}^{o} \quad \forall(i, j) \in A, \quad \forall o \in O \\
y_{i j} \leq \sum_{\forall o \in O} x_{i j}^{o} \quad \forall(i, j) \in A \\
\sum_{\forall j \in V_{i}^{+}} x_{j i}^{o}-\sum_{\forall k \in V_{i}^{-}} x_{i k}^{o}=\left\{\begin{aligned}
-1 & \text { if } i=s^{o} \\
0 & \text { if } i \in V \backslash\left\{s^{o}, t^{o}\right\} \\
1 & \text { if } i=t^{o}
\end{aligned}\right. \\
\text { for all nodes } i \in V \text { and commodities o } \\
y 1_{j i}=\sum_{\forall k \in V_{i}^{-}} y 1_{i k} \quad \forall i \in V \backslash V_{E}, \forall j \in V_{i}^{+} \\
y 2_{j i}=\sum_{\forall k \in V_{i}^{-}} y 2_{i k} \quad \forall i \in V \backslash V_{E}, \forall j \in V_{i}^{+}
\end{gathered}
$$




$$
\begin{array}{cl}
y 2_{j i}=\sum_{\forall k \in V_{i}^{-}} y 1_{i k} \quad & \forall i \in V_{E}, \forall j \in V_{i}^{+} \\
\sum_{\forall(i, j) \in l}\left(y 1_{i j}+y 2_{i j}\right) \leq 1 & \forall l \in L
\end{array}
$$

Variables:

$$
\begin{aligned}
x_{w}^{o} \in\{0,1\} & \forall(i, j) \in A, \quad \forall o \in O \\
y 1_{i j} \in\{0,1\} & \forall(i, j) \in A \\
y 2_{i j} \in\{0,1\} & \forall(i, j) \in A
\end{aligned}
$$

Here constraint (52) state that each working wavelength-path is continuous between its electrical terminations, constraint (53) states the same for protection wavelength-paths, while constraint (54) states, that where a working wavelength-path is terminated, there must start a protection wavelength-path. Constraint (55) guarantees that the working wavelength-path and protection wavelength-path must be disjoint.

Node Disjoint Case. To obtain protection path which is nodedisjoint of the working one we need to substitute constraint (55) by the following one.

$$
\sum_{\forall i \in n \backslash V_{E}} \sum_{\forall j \in A_{i}^{+}}\left(y 1_{i j}+y 2_{i j}\right) \leq 1 \quad \forall n \in N
$$

\section{COMMENTS ON THE PROBLEM}

In paper [2] the authors present different ways of formulating the problem for both single-hop and multihop lightpaths referred to as Wavelength Path (WP) and Virtual Wavelength-Path (VWP) respectively. Among other methods they also use ILP. The drawback of the VWP formulation using ILP (and also of other methods applied to VWP) is that they implicitly assume pure electrical nodes, where not only electrical space-switching but also time-switching, i.e., re-multiplexing has to be performed. This approach degrades the Wavelength Routing network to a network employing WDM links and therefore it requires electrical (e.g., ATM) switches of large capacities increasing the costs and deteriorating the performance. The advantage of the method proposed in this paper is that it can differentiate various nodes with flexible functionality.

In paper [7] also a graph-model has been used but vertices are arranged in a matrix like grid, and edges representing lightpaths afterwards. Although the method is advantageous because of the applicability of fast shortest path algorithms, it does not take into account 
different node-types except the electrical switches. Furthermore, the method is used for routing of lightpaths only, not for configuration, i.e., simultaneous routing of all traffic demands.

The bin-packing problem, where we want to pack objects of different size into bins (of equal size) in optimal way is NP-hard. Its generalisation for the multidimensional case is called vector-packing. If the wavelengthchannel capacities over all links are considered to be "bins" which form a vector indexed by links and we want to "pack" them optimally by traffic streams of different demands (different "objects"), we would have the same problem. However, our problem is even more complex, since there is "interaction" between "bins", because loading one bin will induce loading one of the neighbouring bins. However, these relations change dynamically, since if two neighbouring bins are filled by an object, it will avoid filling some other bins. For the above reasons this method is expected to be also NP-hard.

\section{SOLUTION ALTERNATIVES}

The presented network model allows many methods for solving the above formulated problem. The alternatives include, but are not limited to:

- ILP Solvers

- Relaxing ILP to LP and then rounding the variables.

- Generally applicable heuristics for global optimisation directly applied for 0-1 programming (e.g., Simulated Annealing (SA), Genetic Algorithm (GA), Threshold Accepting (TA), Tabu Search (TS), Go with the Winners (GW), etc.).

- Generally applicable heuristics for global optimisation (SA, GA, TA, TS, GW,...) applied through specially matched models.

- Heuristic Algorithms based on decomposition.

Solving the problem by any available ILP solver (e.g., LP_ SOLVE or CPLEX) will be possible for very small networks only. The reason is that the number of both, variables and constraints will grow by increasing the size of the network and this will result in exponential growth of the al ternatives to be investigated by ILP software. Although ILP solvers give global optimum, they do not solve problems of larger scale. There are alternatives.

It is possible to relax integrality condition, i.e., to use continuous variables bounded by the closed interval $[0,1]$ inserted of binary ones. 
In this case the result can be obtained in shorter time even for problems of large scale, however, rounding of variables can be needed.

Another alternative is to apply directly to ILP formulation randomised methods, e.g., Simulated Annealing, Genetic Algorithm or Tabu Search - all using binary encoding. All constraints are evaluated, and if violated a penalty term is added to the objective. The penalty term for each constraint is a function of the penalty violation. Although different functions were tried out, ranging from linear to those which punish large deviations from the constraint more strictly, the method does not give expected results. Fine tuning of coefficients in the linear combination of the objective and penalty terms was needed. However, it has happened sometimes, that some traffic demands were not satisfied $(x, y$ and $z$ variables were 0 ), but non of the constraints was violated.

Based on the above formulation even more sophisticated heuristic approximation methods can be used. The idea is to exclude a part of the state-space which is not of interest, instead of using penalty term as a means of obeying constraints.

The decomposition according to the node-pairs appeared to be very promising as well. This is approximation only, but a very fast one. In this case the demands are routed one-by-one.

\section{CONCLUSION}

In this paper the general model for DWDM networks is proposed which enables the ILP formulation of the static RWA problem including traffic grooming and protection at either electrical or WDM layer. ILP formulation is proposed for both, directed and undirected graph models.

In static case this approach allows configuration of combined MPLSMP $\lambda$ S (e.g., GMPLS) networks. This approach can be used for dynamic RWA as well, furthermore it can be combined by routing techniques like OSPF, OMP-OSPF or PNNI.

The advantage of our approach is that it optimises jointly the electrical layer and the underlying wavelength or fiber system, it includes various protection mechanisms, and it supports traffic grooming whenever the number of available wavelengths is not sufficient, but certain wavelength channels have some spare capacity.

\section{ACKNOWLEDGEMENTS}

This work has been done in the research co-operation framework between Ericsson and the High-Speed Networks Laboratory (HSNLab) at the Department of Telecommunications and Telematics, Budapest Uni- 
versity of Technology and Economics. The authors are grateful to Miklós Boda (Ericsson) and Tamás Henk (HSNLab) for their support. 


\section{References}

[1] P.E. Green, "Optical Networking Update", IEEE Journal on Selected Areas in Communications, vol. 14, no. 5, pp. 764-779, June 1996

[2] N. Wauters, P. Demeester, "Design of the Optical Path Layer in Multiwavelength Cross-Connected Networks", IEEE Journal on Selected Areas in Communications, vol. 14, no. 5, pp. 881-892, June 1996

[3] Z. Zhang, A.S. Acampora, "A Heuristic Wavelength Assignment Algorithm for Multihop WDM Networks with Wavelength Routing and Wavelength Re-Use" Transactions on IEEE Networking, vol. 3, no. 3, pp. 281-288, June 1995

[4] R. Ramaswami, K.N. Sivarajan, "Routing and Wavelength Assignment in All-Optical Networks", IEEE Transactions on Networking, vol. 3, no. 5, pp. 489-500, Oct. 1995

[5] R. Ramaswami, K.N. Sivarajan, "Design of Logical Topologies for Wavelength-Routed Optical Networks", IEEE Journal on Selected Areas in Communications, vol. 14, no. 5, pp. 840-851, June 1996

[6] D. Banerjee, B. Mukherjee, "A Practical Approach for Routing and Wavelength Assignment in Large Wavelength-Routed Optical Networks", IEEE Journal on Selected Areas in Communications, vol. 14, no. 5, pp. 903-908, June 1996

[7] I. Chlamtac, A. Faragó, T. Zhang, "Lightpath (Wavelength) Routing in Large WDM Networks" IEEE Journal on Selected Areas in Communications, vol. 14, no. 5, pp. 909-913, June 1996

[8] B. Mukherjee, D. Banerjee, S. Ramamurthy, A. Mukherjee, "Some Principles for Designing a Wide-Area WDM Optical Network", IEEE Transactions on Networking, vol. 4, no. 5, pp. 684-696, Oct. 1996 
[9] H. Harai, M. Murata, H. Miyahara, "Performance of All-Optical Networks with Limited-Range Wavelength Conversion" ICC'97 IEEE International Conference on Communications, Montréal, June 1997

[10] M. Garnot, M. Sotom, F. Masetti, "Routing Strategies for Optical Paths in WDM Networks", ICC'97 - IEEE International Conference on Communications, Montréal, June 1997

[11] J. Armitage, O. Crochat, J.-Y. Le Boudec, "Design of a Survivable WDM Photonic Network", INFOCOM 97, Kobe, April 1997

[12] L. Berthelon et.al., "Towards photonic networking: experimental demonstration of a reconfigurable survivable WDM ring network", GLOBCOM, London, November 1996

[13] K. Bala, G. Ellinas, M. Post, "Towarwds Hitless Reconfiguration in WDM Optical Networks for ATM Transport", GLOBCOM, London, November 1996

[14] E. Karasan, E. Ayanoglu, "Effects of Wavelength Routing and Selection Algorithms on Wavelength Conversion Gain in WDM Networks", GLOBCOM, London, November 1996

[15] S. Banerjee, C. Chen, "Design of Wavelength-Routed Optical Networks for Circuit Switched Traffic", GLOBCOM, London, November 1996

[16] D. Banerjee, B. Mukherjee, "Wavelength-Routed Optical Networks: Linear Formulation, Resource Budgeting Tradeoffs and a Reconfiguration Study", INFOCOM 97, Kobe, April 1997

[17] J.-F. P. Labourdette, "Performance Impact of Partial Reconfiguration on Multihop Lightwave Networks", IEEE Transactions on Networking, vol. 5, no. 3, pp. 351-358, June 1997

[18] T. Cinkler, "Heuristic Algorithms for Configuration of the ATMLayer over Optical Networks" ICC'97 - IEEE International Conference on Communications, Montréal, June 1997

[19] R.M. Krishnaswamy, K.N. Sivarajan, "Design of Logical Topologies: a Linear Formulation for Wavelength Routed Optical Networks with no Wavelength Changers", IEEE INFOCOM'98, March 1998

[20] S. Johansson, "Transport Network Involving a Reconfigurable WDM Network Layer - A European Demonstration", IEEE Journal on Lightwave Technology, vol.14, no.6, pp. 1341-1348, June 1996 
[21] T. Cinkler, R.S. Castro, S. Johansson, "Configuration and Reconfiguration of WDM Networks", NOC'98 - Conference on Networks and Optical Communications, Manchester, June 1998

[22] M.R. Garey, D.S. Johnson, "Computers and Intractability - A Guide to the Theory of NP-completeness", W.H. Freeman and Co., New York, 1979

[23] I. Chlamtac, A. Ganz, G. Karmi, "Lightpath Communications: An Approach to High Bandwidth Optical WANs", IEEE Transactions on Communications, vol. 40, no.7, pp. 1171-1182, July 1992

[24] R.K. Ahuja, T.L. Magnanti, J.B. Orlin, "Network Flows, Theory, Algorithms and Applications", Prentice-Hall, 1993 